\title{
La convergencia conceptual como escenario de investigación en comunicación, diseño gráfico, publicidad y mercadeo*
}

\author{
Janneth Arley Palacios Chavarro** \\ Patricia Lora León ${ }^{* * *}$ \\ Recibido: 2017-05-16 Enviado a pares: 2017-05-25 \\ Aprobado por pares: 2017-07-18 Aceptado: 2017-07-25 \\ DOI: 10.22395/angr.v16n31a9
}

\begin{abstract}
Resumen
Frente a los diferentes debates sobre la pérdida de identidad de la investigación en comunicación y sus campos conexos, este artículo plantea escenarios que reconozcan la convergencia de categorías comunes entre campos como la comunicación, la publicidad, el diseño gráfico y el mercadeo. Para esto, se propone la reflexión desde tres perspectivas: la interdisciplinariedad conceptual, los conceptos nómadas y los conceptos viajeros.
\end{abstract}

Esta investigación de tipo analítico-descriptiva y de carácter mixto, presenta posteriormente, un grupo de categorías convergentes que son el resultado de la revisión de la producción académica y científica incluida en 313 documentos entre 2012 y 2016 de la Facultad de Ciencias de la Comunicación de la Fundación Universitaria Los Libertadores (Colombia), en los que se evidenció empíricamente que dichas categorías responden a la interdisciplinariedad conceptual y a la tesis propuesta desde los conceptos "nómadas" o "viajeros".

Se demostró que los conceptos ya no son de exclusividad de una disciplina, sino que al resignificarse y migrar, amplían el horizonte de las disciplinas, las enriquecen y permiten una mirada más holística de la realidad. Se concluyó que la interdisciplinariedad conceptual plantea una perspectiva para atravesar los límites de las disciplinas y repensar la investigación en comunicación en función de sus diferentes conexiones con otros campos y saberes.

Palabras clave: convergencia conceptual; conceptos viajeros; convergencias en comunicación; conceptos nómadas; interdisciplinariedad conceptual en comunicación; investigación en comunicación; creatividad en comunicación.

Este artículo es resultado del proyecto de investigación titulado "Investigar la investigación en la Facultad de Ciencias de la Comunicación", vinculado al grupo de investigación Comunicación, Cultura y Tecnología, de la Facultad de Ciencias de la Comunicación de la Fundación Universitaria Los Libertadores, Bogotá, Colombia.

** Comunicadora social, magíster en Administración. Profesora e investigadora de la Facultad de Ciencias de la Comunicación. Fundación Universitaria Los Libertadores, Bogotá, Colombia. japalaciosc@libertadores.edu.co

*** Comunicadora social, magíster en investigación social interdisciplinaria y Doctora en Ciencias Sociales. Profesora e Investigadora de la Facultad de Ciencias de la Comunicación. Fundación Universitaria Los Libertadores, Bogotá, Colombia.mploral@libertadores.edu.co 


\title{
Conceptual convergence as a research scenario in communication, graphic design, advertising, and marketing
}

\begin{abstract}
Before different debates on the loss of identity of communication research and its related fields, this article proposes scenarios that recognize the convergence of common categories among fields such as communication, advertising, graphic design, and marketing. For this purpose, reflection is proposed from three perspectives: conceptual interdisciplinary, nomadic concepts, and traveling concepts.

This analytical-descriptive and mixed nature research presents, subsequently, a group of convergent categories that are the result of the review of the academic and scientific production included in 313 documents between 2012 and 2016 of the School of Communication Sciences of Fundación Universitaria Los Libertadores (Colombia), in which it was empirically demonstrated that said categories respond to the conceptual interdisciplinary and to the thesis proposed from the concepts "nomads" or "travelers".

It was shown that the concepts are no longer exclusive to a discipline, but that by re-signifying and migrating, they broaden the horizon of the disciplines, enrich them and allow a more holistic view of reality. It was concluded that conceptual interdisciplinary raises a perspective to cross the limits of disciplines and rethink communication research in terms of its different connections with other fields and knowledge.
\end{abstract}

Keywords: conceptual convergence; traveling concepts; convergences in communication; nomadic concepts; conceptual interdisciplinary in communication; communication research; creativity in communication.

\section{A convergência conceitual como cenário de pesquisa em comunicação, desenho gráfico, publicidade e marketing}

\begin{abstract}
Resumo
Ante os diferentes debates sobre a perda de identidade da pesquisa em comunicação e seus campos conexos, este artigo apresenta cenários que reconheçam a convergência de categorias comuns entre campos como a comunicação, a publicidade, o desenho gráfico e o marketing. Para isso, propõe-se a reflexão sob três perspectivas: a interdisciplinaridade conceitual, os conceitos nômades e os conceitos viajantes. Esta pesquisa de tipo analíticodescritivo e de caráter misto apresenta, em seguida, um grupo de categorias convergentes que são o resultado da revisão da produção acadêmica e científica incluída em 313 documentos entre 2012 e 2016 da Faculdade de Ciências da Comunicação da Fundación Universitaria Los Libertadores (Colômbia), nos quais se evidenciou empiricamente que essas categorias respondem à interdisciplinaridade conceitual e à tese proposta a partir dos conceitos "nômades" ou "viajantes". Demonstrou-se que os conceitos já não são de exclusividade de uma disciplina, mas sim que, ao se ressignificarem e migrarem, ampliam o horizonte das disciplinas, enriquecem-nas e permitem um olhar mais holístico da realidade. Concluiu-se que a interdisciplinaridade conceitual sugere uma perspectiva para ultrapassar os limites das disciplinas e repensar a pesquisa em comunicação em função de suas diferentes conexões com outros campos e saberes.

Palavras-chave: conceitos nômades; conceitos viajantes; convergência conceitual; convergências em comunicação; criatividade em comunicação; interdisciplinaridade conceitual em comunicação; pesquisa em comunicação.
\end{abstract}




\section{Introducción}

El presente artículo toma como punto de partida una relectura de las posibilidades de investigación en comunicación desde la convergencia conceptual y la interdisciplinariedad. Para ello, busca repensar dicha investigación considerando la naturaleza igualmente convergente en el ámbito académico y profesional entre los campos de la comunicación, la publicidad, el mercadeo y el diseño gráfico'.

Parte del supuesto de que la literatura existente en este ámbito centra su preocu pación en el debate sobre las relaciones que estructuran y constituyen el campo de la comunicación a partir del "diálogo interdisciplinario" (Aguirre, Anaya, Laurencio, E Casco López, 2013), intrínseco en la relación que la investigación en comunicación ha establecido con los marcos epistemológicos y metodológicos de otras disciplinas (Piñuel, 2009), bien desde la dimensión sistémica de la comunicación que comparte con disciplinas como la física, la biología y la etología, o desde la dimensión del comportamiento humano que comparte con la psicología y la psiquiatría; o de manera más específica, con las ciencias sociales y humanas en su análisis sobre las relaciones sociales y culturales, entre otras (Aguirre, Anaya, Laurencio, E Casco López, 2013).

No obstante, el carácter interdisciplinario ya enunciado de la comunicación ha conllevado serias implicaciones en la comprensión de su naturaleza, objeto y contenidos, generado importantes debates de orden académico e investigativo que aún permanecen abiertos (Sierra-Gutiérrez, 2016). El contexto general de la investigación en comunicación evidencia la fuerte influencia de otras áreas de conocimiento, agudizando aún más el debate. Así, con la sociología o la antropología, la comunicación ha establecido modelos teóricos como el positivismo con todas sus vertientes o el análisis materialista dialéctico con sus perspectivas de análisis, en donde la comunicación encuentra elementos con los que construye su discurso y propone líneas de investigación (Piñuel, 2009). Por su parte, el funcionalismo de corte positivista encuentra en Norteamérica el terreno abonado para la producción en campos como la comunicación, la publicidad y el diseño; por el otro lado, de la corriente materialista dialéctica encuentra puntos de anclaje en las corrientes estructuralista o en la teoría crítica que han generado interesantes análisis en comunicación y el diseño gráfico .

Esta situación muestra entonces que en el campo de la comunicación coexiste una amplia variedad de objetos de estudio, de enfoques teóricos (Igartua, 2012), de plantea-

\footnotetext{
Es notable en el nivel formativo, cómo diversas escuelas o facultades de comunicación social en Colombia y el mundo, incorporan diversos programas relacionados con estos campos. Acá se toma como referencia la Facultad de Ciencias de la Comunicación de la Fundación Universitaria Los Libertadores, que es la Institución que financia el proyecto. Aunque es posible citar otras Facultades de Comunicación Social que, en Colombia, incluyen en su oferta académica convergencias similares, como es el caso de la Facultad de Mercadeo, Comunicación y Artes del Politécnico Grancolombiano (Diseño de Modas, Mercadeo y Publicidad, Diseño Industrial. Comunicación Social Periodismo, Diseño Gráfico, entre otros), la Facultad de Ciencias de la Comunicación de la Universidad Autónoma de Occidente (Comunicación Social. Periodismo, Diseño de la Comunicación Gráfica, Comunicación Publicitaria y Cine y Comunicación Digital) o la Facultad de Comunicación y Lenguaje, con sus programas de pregrado en Comunicación Social, Licenciatura en Lenguas Modernas y Ciencia de la Información.
} 
mientos y de temas con poca conexión entre ellos (Otero, 2010), lo que hace que para algunos autores "los estudios sobre los fenómenos de la comunicación conformen un conjunto intelectual fragmentado y disperso, una variedad de planteamientos que no dialogan entre sí, que con frecuencia se excluyen o se ignoran unos a otros y que permanecen aislados" (Otero, 2010, pág. 8). Este panorama del estado de la investigación en comunicación, estudiado ampliamente (Berelson² (1959) citado por Arias (2011); Craig, 1999; Krippendorf, 1994; Piñuel, 2007; Donsbach, 2012; Igartua, 2012; Vidales, 2015; De San Eugenio \& Silva, 2014, entre otros), plantea no solo la ausencia de reflexión sobre sus posibilidades de interdisciplinariedad, sino que muestra su incapacidad de complementarse a sí misma con las propias especialidades a las que ha dado origen.

Por ello, encontrar otras lecturas que permitan dar pistas sobre la complejidad que representa el campo comunicativo se hace cada vez más relevante. Una de esas posibilidades es a partir de identificar y reconocer intereses comunes en torno a escenarios, categorías, conceptos y objetos de estudio; en otras palabras, puntos de convergencia pensados no desde las fronteras disciplinares, sino desde la convergencia conceptual. De esta manera,

[...] El potencial de la comunicación en tanto lugar de convergencias/ divergencias tiene que ver con las posibilidades de reconstrucciones y emergencias de saberes que, de otra forma, permanecerían eclipsados (...). Es claro, hoy por hoy, que ese descenso áspero, a los procesos de construcción(es) de sentido(s) que se dan a lo largo y ancho del andamiaje social, es lo que hoy nos permite, por ejemplo, reinventar los roles propios del proceso comunicativo y pensar en nuevas formas de empoderamiento simbólico que desembocan, sin más, en conceptos que ahora pueblan las coordenadas del campo, como, por ejemplo, convergencia y transmedialidad (Roncallo-Dow, 2013).

Así, a diferencia de las perspectivas anteriores, este artículo plantea otros escenarios de investigación que reconozcan la convergencia de las categorías comunes de campos profesionales y académicos inscritos al ámbito de las ciencias de la comunicación tales como la publicidad, el diseño gráfico y el mercadeo. Para ello, se retoman los postulados de autores como Tamayo (2003), Oncina, Miravet \& Vizcaíno (2014), Vásquez-Alonso \& Manassero-Mas (2017) y Bal (2002), que sugieren otras lecturas de la interdisciplinariedad y colocan los conceptos convergentes entre las disciplinas como un nuevo escenario para pensar otros horizontes epistemológicos y metodológicos.

Consecuente con ello, se propone desde la "interdisciplinariedad conceptual" (Tamayo, 2003), la dinámica de los "conceptos nómadas" (Vázquez-Alonso \& ManasseroMas, 2017); (Oncina, Miravet, E Vizcaíno, 2014) y desde la dimensión metodológica de los "conceptos viajeros" (Bal, 2002), una reflexión sobre la convergencia conceptual y cómo dicha convergencia se constituye en un nuevo escenario para pensar la investigación desde las prácticas trasversales de diversos campos comprometidos en el ámbito de

Desde más de medio siglo atrás, en su trabajo sobre "El estado de la investigación en comunicación" Bernald Berelson, ya había abierto el debate al plantear la muerte del campo de la comunicación (Arias, 2011). 
las ciencias de la comunicación. Esta propuesta, ya iniciada para pensar otros campos como los estudios sobre la cultura, hizo emerger la necesidad de reconocer la pluralidad en las aproximaciones a la comprensión de la cultura y su configuración epistemológica construida desde saberes colectivos (Neumann \& Nünning, 2012). Teniendo en cuenta lo anterior, proponemos un camino para considerar el potencial de los conceptos y su interdisciplinariedad en los estudios en comunicación.

Finalmente, se retoma en este contexto la reflexión desde los espacios de convergencia conceptual identificados en el Proyecto de Investigación "Investigar la investigación" de la Facultad de Ciencias de la Comunicación de la Fundación Universitaria Los Libertadores. En este orden de ideas, se presenta un grupo de categorías convergentes en los campos de la comunicación, la publicidad, el mercadeo y el diseño gráfico, identificadas a partir de los procesos de auto reflexión de los docentes e investigadores de la Facultad (Palacios, Lora, Cárdenas, \& Guillermo, 2016) y que son validadas con el análisis de la producción científica materializada en proyectos de investigación y en artículos en revistas indexadas, entre otros. A este respecto se destaca cómo la categoría "creatividad" y su ámbito de aplicación, constituye un escenario de convergencia conceptual clave para la investigación el ámbito de la comunicación.

\section{Pensando la interdisciplinariedad a partir de la convergencia conceptual}

Diferentes perspectivas sobre el estudio de la comunicación colocan la interdisciplinariedad como la principal evidencia de la ausencia de un campo disciplinar con una reflexión epistemológica, dominio teórico y metodologías propias. El diálogo recurrente con las ciencias sociales y humanas, por ejemplo, determina, en muchos casos, que la comunicación no sea clasificada como ciencia, mientras que desde el surgimiento de la comunicología como "ciencia interdisciplinaria que estudia la comunicación en sus diferentes medios, técnicas y sistemas" (RAE, 1992 citado por Galindo-Cáceres, 2013) o "Ciencia de carácter interdisciplinario que estudia los sistemas de comunicación humana y sus medios" (RAE, 2004 citado por Galindo-Cáceres, 2013), se plantea una escenario que valora de manera positiva la convergencia disciplinar en torno a un dominio de estudio (Aguirre, Anaya, Laurencio, E Casco López, 2013, pág. 306) y que implica:

Una encrucijada inter y transdisciplinar, dentro de las ciencias sociales y humanidades, lo que lo hace un reto aún mayor, en la medida en que exige de cada uno -dependiendo de los objetos más específicos de investigación- el desarrollo de un amplio espectro de capacidades teóricas y metodológicas. Fuentes (1997) citado por (Aguirre, Anaya, Laurencio, E Casco López, 2013, pág. 307).

En este sentido, se afirma que la interdisciplinariedad se origina al "tratar de resolver problemas complejos que desbordan la matriz disciplinar, y cuya solución requiere importar paradigmas o conceptos de otras disciplinas (Vázquez-Alonso \& Manassero-Mas, 2017). Por tanto, la interdisciplinariedad en comunicación es entendida entonces, desde el punto de vista de la comunicología, como una ciencia capaz de analizar diversos objetos y sujetos de estudios, en diálogo con diversos presupuestos epistemológicos y metodo- 
logías de otras disciplinas (Aguirre et al., 2013) y sería, en este sentido, el resultado de "pensar la comunicación" en un intento por auto reflexionarse (Galindo-Cáceres, 2013). Esto implica, de alguna manera, reconocer la interdisciplinariedad como potencia y no como obstáculo, planteando la comunicación como un escenario interdisciplinar, tejido en la convergencia de diversos procesos y realidades que atraviesan diversos campos disciplinares, o como se propone en el presente análisis, tejido, a su vez, por la convergencia de conceptos y categorías propias y de otros campos del conocimiento. Frente a esta posibilidad, se rescata que,

El recurso teórico-retórico a la interdisciplinariedad exige, de entrada, el abandono de los prejuicios positivistas y reclama una mirada más amplia. La interdisciplinariedad no puede ser pensada como un lugar de conjunción de disciplinas sino como un punto-génesis de poiesis en el que se dan las condiciones para la emergencia de nuevos conocimientos, híbridos. Esta hibridación se abre cada vez más camino hoy en día, cuando la especialización absoluta cae en el anacronismo. La fragmentación de cada disciplina deja lagunas entre las especialidades, que se suman a las que produce la división de las ciencias sociales en disciplinas establecidas (Roncallo-Dow, 2013, pág. 278)

Desde otro punto de vista, para Tamayo (2003) la ciencia investiga aspectos de la realidad para comunicar sus hallazgos. En este sentido, los conceptos son fundamentales para el proceso de producción y comunicación de la ciencia en la medida en que son abstracciones dentro de un marco de referencia específico del conocimiento. La interdisciplinariedad, para el autor, busca incorporar los resultados de las diferentes disciplinas y para ello recurre a "esquemas conceptuales de análisis", lo que permite de hecho la comparación y enjuiciamiento de los mismos. Por ello, la interdisciplinariedad, como un factor positivo en el avance y progreso de las diferentes ciencias y como metodología de investigación científica, no puede ser considerada, según el autor, como un proceso unívoco. En virtud de esto, propone diferentes tipos de interdisciplinariedad ${ }^{3}$, entre ellas, la interdisciplinariedad conceptual, que hace referencia a "cuando a partir de un concepto de carácter genérico, independiente de una disciplina específica, hacemos claridad de fenómenos presentados en la realidad y que pueden ser estudiados por diversas disciplinas" (Tamayo, 2003, pág. 81).

Resulta interesante en la clasificación de Tamayo encontrar cómo los conceptos hacen posibles otras formas de interdisciplinariedad. Según la definición de "interdisciplinariedad conceptual" propuesta, "conceptos genéricos" se convierten en herramientas para el estudio de la realidad y permiten no solo el diálogo entre disciplinas sino "hacer claridad" frente a los fenómenos estudiados. En el caso particular del diseño, la publicidad y el mercadeo, o inclusive frente la ausencia de un consenso sobre el carácter de ciencia de la comunicación como fue citado anteriormente, nos deparamos frente a campos académicos y profesionales que no cuentan con el estatus de ciencia desde el planteamiento de la disciplinariedad, pero que desde la perspectiva de escenarios como el de la comunicología o de la "interdisciplinariedad conceptual" recuperamos no solo objetos y

Instrumental, auxiliar, limítrofe, estructural, metodológica, entre otras. 
sujetos de estudio comunes sino conceptos convergentes que permiten una nueva mirada sobre los propios fenómenos a analizar.

Lo que hasta aquí se ha planteado como un escenario de convergencia conceptual, que hace posible un cierto tipo de interdisciplinariedad, es problematizado desde la postura de otros autores como la existencia misma no solo de la convergencia, sino de la "migración" o movilidad de los conceptos que determina no apenas su función como herramientas metodológicas de análisis de la realidad sino como manifestaciones de vitalidad y dinamismo, propios de los conceptos más allá de las fronteras disciplinares. Así, "el concepto nómada no modifica su naturaleza constitutiva, pero innova la orientación y el desarrollo de la disciplina receptora, pues le aporta metáforas y elementos extrínsecos y nuevos" (Vázquez-Alonso E Manassero-Mas, 2017, pág. 28).

Sumado a lo anterior, Müller (2013) citado por Oncina, Miravet E Vizcaíno (2014), considera, de hecho, la existencia de "conceptos interdisciplinares" entendidos como "aquellos que se usan en muchas disciplinas, se resisten a agotarse en una definición, poseen una sobreabundancia constitutiva de significados que los hacen tan controvertidos como productivos para la generación de nuevos discursos" (pág. 9). Para el autor, dichos conceptos se encuentran en el cruce entre disciplinas, las atraviesan transversalmente e incluso proceden de un tiempo anterior a la división disciplinar. También cumplen una "función catalizadora" entre diferentes ámbitos del saber al derribar barreras disciplinares y culturales (Oncina, Miravet, E Vizcaíno, 2014).

Oncina et al., (2014) recuperan esta dimensión de los "conceptos interdisciplinares" de Müller, y plantean la importancia de una "historia conceptual interdisciplinar", capaz de dar cuenta de una dimensión de los conceptos, no cómo objetos estáticos, "sino como cúmulo de sedimentos o capas significativas que no cesan de estar en movimiento" (pág. 11). Dicha historia se reconstruye para el autor a partir de la aproximación a estos "conceptos nómadas" o conceptos viajeros que cruzan fronteras políticas, lingüísticas, temporales y disciplinares:

La mayoría de los conceptos han sido tallados y pulimentados diversamente, según las épocas, las tradiciones y los ámbitos de aplicación, y este nomadismo ha dado lugar a transformaciones, reconversiones, metamorfosis o simples matizaciones que han enriquecido la historia del pensamiento y la capacidad de obrar del ser humano (Oncina, Miravet, E Vizcaíno, 2014, pág. 12).

Por otro lado, desde el análisis de las humanidades, Bal (2002) plantea también la existencia de "conceptos viajeros", entendidos desde su perspectiva como categorías transversales y en permanente transformación, que "migran", se transforman o cambian a través de diferentes disciplinas sociales y humanas adquiriendo múltiples sentidos y usos desde lo teórico y lo metodológico.

Para la autora, los conceptos son herramientas de la intersubjetividad que, a su vez, facilitan la conversación posibilitando un lenguaje común. Si bien se les considera una representación abstracta del objeto, para Bal, declarar que algo es una "imagen", una 
"metáfora", una "historia" o lo que se quiera a través de un concepto implica también una serie de opciones interpretativas que no son posibles de ocultar. En tal sentido, considera que los conceptos no están fijos, pues "viajan" -entre disciplinas, eruditos, lugares, tiempos, personas e incluso comunidades separadas geográficamente- transformando de esa manera su significado, alcance y valor operativo y haciéndolos flexibles. Algunos ejemplos de conceptos viajeros son "clase, raza, género" (Olson, 2012), "sostenibilidad" (Knauf, 2014) e "hibridación" (Bal, 2002).

De acuerdo con la autora, los conceptos necesitan ser explícitos, claros y definidos para que las personas los puedan adoptar y utilizar, y para que puedan servir para "ayudar a articular un cierto entendimiento", a pesar de que estos sean flexibles y viajen en el tiempo.

Pero los conceptos solo pueden realizar esta tarea, la tarea metodológica que anteriormente realizaban las tradiciones disciplinares, con una condición: que se sometan a escrutinio no solo mediante su aplicación a los objetos culturales que examinan, sino a través de la confrontación con ellos, ya que los mismos son sensibles al cambio y sirven para revelar diferencias históricas y culturales (Bal, 2002, págs. 30-31).

A través de su reflexión sobre los conceptos "viajeros", Bal propone una alternativa metodológica para abordar la investigación desde los conceptos, es decir, desde la observación detallada de las relaciones, usos y significaciones de esos conceptos entre diferentes campos del conocimiento y en el contexto de realidades sociales y culturales particulares. Invita, por lo tanto, a pensar sobre lo que los conceptos "pueden hacer", problematizando su naturaleza representacional y estática para indagar sobre su mutabilidad (su capacidad de "viajar") y sobre las relaciones que esos conceptos operan en su interacción con artefactos y contextos culturales específicos.

El cambio de metodología que estoy proponiendo, se basa en una relación particular entre sujeto y objeto, una relación que no se conforma en base a una oposición vertical y binaria entre los dos. En lugar de ello, esta relación tiene como modelo la interacción, en el sentido que el término tiene en "interactividad". La razón por la que tomarse en serio los conceptos resulta provechoso para todos los campos académicos, pero especialmente las humanidades, que cuentan con muy pocas tradiciones aglutinadoras, es esta potencial interactividad y no una obsesión con el uso "correcto" de las palabras (Bal, 2002, pp. 30-31).

Si los conceptos son "tallados" según las épocas y tradiciones, pero también por sus "ámbitos de aplicación", siguiendo los planteamientos de Oncina et al., (2014) sobre los "conceptos nómadas", o se constituyen en una "alternativa metodológica" a partir de la observación detallada de sus relaciones, usos y significaciones como lo plantea Bal (2002), para el caso de las humanidades, entonces la convergencia conceptual puede constituirse en el ámbito de los campos profesionales y académicos que integran las denominadas Ciencias de la Comunicación (entre ellos la comunicación social, la publicidad, el mercadeo y el diseño gráfico) en una oportunidad para identificar nuevos escenarios de investigación que indaguen, no solo sobre su utilidad teórica o pertenencia a un cuerpo teórico o disciplinar específico, sino sobre lo que "pueden hacer" esos conceptos y desde 
lo que esos conceptos representan y aportan a los campos académicos y profesionales ya enunciados, en su función de resolver problemas comunes de la sociedad.

\section{La convergencia conceptual en las ciencias de la comunicación}

Castellanos (2015), en un ejercicio enunciativo, ofrece un conjunto de elementos comunes y convergentes a los campos de la comunicación social, el diseño, la publicidad y el mercadeo desde diferentes ópticas y horizontes en la construcción de sus cuerpos teóricos. Destaca no solo el interés de los diferentes campos por "entender la comunicación como fenómeno y práctica dentro de un contexto determinado", sino sus problemas comunes a resolver en diferentes sectores de la sociedad y las lógicas y rutinas productivas comunes vinculadas a las dinámicas de producción, circulación y consumo, el abordaje desde la cultura y la noción de tecnología asociada prioritariamente desde la mirada técnica.

Evidencia también en este contexto, la convergencia de categorías como "imágenes, visualidades, discursividades, palabra, retórica y la riqueza dentro de la construcción de cada uno de los lenguajes (gráfico, publicitario, periodístico, etc.)", el fenómeno del consumo y sus prácticas, la discusión por las estéticas y las narrativas (relatos y mitologías como en el caso publicitario), así como de otro grupo de nociones cómo: "comunicación, información, cultura, lenguaje, soportes, representación, persuasión, percepción, significación, mediación, medios de comunicación, productos de comunicación, contexto sociocultural, globalización, modernidad, entre otros" (Castellanos, 2015).

Desde otra área de conocimiento, específicamente desde la didáctica de las ciencias (DC), Vázquez-Alonso \& Manassero-Mas (2017) analizando líneas básicas de congresos internacionales encontraron un conjunto de conceptos interdisciplinares nómadas, entre ellos, los más relevantes fueron: evaluación, competencias y el más importante: práctica que incluye diversos tipos como práctica educativa, prácticas de aprendizaje, prácticas de laboratorio, práctica científica, etc. Los autores concluyeron que "las múltiples formas de la práctica educativa dominan los programas de investigación en DC" (pág. 31). Asimismo, realizaron una clasificación de conceptos nómadas asociados a disciplinas fuente. Por ejemplo, de la psicología se toman conceptos como toma de decisiones, creencias y actitudes, entre otros, que en este sentido entrarían a considerarse conceptos nómadas interdisciplinares para la Didáctica de las Ciencias.

De manera complementaria a la propuesta anterior, Vizcarra (2007) durante el período 1943-2005, encontró en los estudios de comunicación algunas tendencias y temas recurrentes como:

[...] historia y estructura de los medios, industrias culturales y economía política de la comunicación, estudios sobre contenidos y audiencias, géneros discursivos, lenguajes, mediaciones de la comunicación, procesos de recepción, comunicación y globalización, arte y medios, expresión y tecnologías de información, prácticas culturales y comunicación, cultura urbana, representaciones sociales, imaginarios e identidades, comunicación social, comunicación y política, comunicación organizacional, enseñanza e investigación de la comunicación y comunicología, entre otros (Vizcarra, 2007, pp. 1-2). 
En estos temas se ha centrado el interés de las investigaciones en el campo de la comunicación, en la región analizada por el autor.

Por otra parte, en 2004, en Francia, otro estudio presentó un conjunto de temas de interés y tendencias en los estudios realizados en esta parte de Europa, identificando cuatro ejes de convergencia: lo económico, lo histórico, la relación con la técnica y los objetos y las significaciones (Lancien, y otros, 2004). Los temas encontrados fueron:

- Medios tradicionales: espacio público y comunicación; economía del sector; recepción y público; análisis del discurso; sociología de contenidos, historia de los medios, usos, profesión.

- Medios nuevos: usos, técnicos, políticas públicas a escala nacional o local, historia de las ciencias y de las técnicas de máquinas de comunicar, derecho y nuevas tecnologías de comunicación y de información, economía y tecnologías de comunicación e información, recepción.

- Cultura y comunicación: modernización técnica y cambios culturales, industrias culturales, representaciones, multiculturalidad, cine documental, imagen, identidad.

- Educación: mutación de los conocimientos como consecuencia de la evolución técnica, redes de difusión de conocimientos especializados, profesiones.

- Comunicación empresarial y organizacional: publicidad, mercadotecnia, reorganización y nuevos modos de gestión frente a las estrategias de comunicación empresarial o de colectividades locales

- Territorio: fomento de recursos territoriales, políticas públicas, la ciudad.

- Arte e hipertexto: estética de la imagen digital, prácticas artísticas en la Internet, historia de las imágenes, museología, música, historia social de la música, literatura e interactividad (Lancien, y otros, 2004).

En este orden de ideas, la convergencia conceptual -ya enunciada por Castellanos-, y planteada en otros estudios, se convierte en una herramienta pertinente para repensar los procesos, espacios, formas de pensar y desarrollar la investigación desde el ámbito de las ciencias de la comunicación, aun cuando en su debate -como ya se ha mencionadose dé una amplia variedad de objetos de estudio -la mayoría provenientes de otras áreas del conocimiento- (Igartua, 2012), de enfoques teóricos, de planteamientos y de temas con poca conexión entre ellos (Otero, 2010), se reafirme una tradición basada en oficios y se mantenga la influencia de perspectivas externas (Castellanos, 2015).

De esta manera, el proyecto "Investigar la investigación en la Facultad de Ciencias de la Comunicación" del cual se desprende este estudio, cuyo propósito fue identificar y analizar las dinámicas y las formas sobre las cuales se están abordando o construyendo los problemas de investigación que se trazan en las aulas y en los diferentes proyectos 
liderados o en los que participa la Facultad a través de sus tres programas (Comunicación Social, Diseño Gráfico y Publicidad y Mercadeo) retoma esta convergencia conceptual como una oportunidad para observar y construir nuevos escenarios de investigación interdisciplinar.

Por ello, siguiendo como punto de referencia las categorías enunciadas anteriormente y sobre la evidencia empírica de la producción de la Facultad durante el periodo 2012 a 2016, a través de sus diferentes proyectos de investigación (artículos, ponencias, libros, material audiovisual, informes, cursos, entre otros), los trabajos de grado elaborados por los estudiantes como requisito para optar a su título profesional, las actividades académicas y científicas, los espacios académicos desarrollados en el plan de estudios y las diferentes producciones de estudiantes y docentes, se identificaron los siguientes (7) grupos de categorías que integran algunos de estos conceptos convergentes a los tres campos, se trata de:

- Producción, circulación y consumo

- Producción de sentido, mediación y cultura

- Lenguajes, estéticas y narrativas

- Herramientas, medios, plataformas y artefactos

- Gestión y estrategias de comunicación

- Creatividad e innovación

- Públicos

Así, fue posible encontrar, en primer lugar, un grupo de conceptos asociados a la mirada de la comunicación, desde la economía política y desde los estudios culturales que permitió una comprensión del proceso comunicativo que, hasta hoy, resulta fundamental para entender y organizar las dinámicas de producción de mensajes y artefactos construidos por los profesionales que participan de la producción cultural y comunicativa en las industrias culturales, del entretenimiento, creativas y en los medios de comunicación en sus más diversas expresiones. Se trata de las categorías de Producción, circulación y consumo, que son consideradas como las etapas que pretenden describir los actores y factores que inciden en las condiciones de producción de mensajes, en la circulación de dichos mensajes y en las representaciones simbólicas y las prácticas vinculadas al consumo (Boni, 2008).

Es importante anotar cómo estos procesos han adquirido nuevas dinámicas a la luz de los nuevos fenómenos comunicativos mediados por plataformas y dispositivos tecnológicos, configurando nuevas prácticas comunicativas: el prosumo, por ejemplo (Zicavo, 2009). Planteamientos desde la "ecología de los medios" explican esta relación "en los sistemas digitales, todos somos parte de él, no hay entorno externo, y cada acto de consumo es igualmente un acto de circulación y de producción" (Vizer $\mathcal{E}$ Carvalho, 2015). 
En este sentido, como apunta Scolari (2008), se asiste a nuevas lógicas del proceso comunicativo que diluyen las fronteras entre la producción y el consumo (prosumidores ${ }^{4}$ ), en cuanto las hipermediaciones proponen nuevos circuitos y canales de distribución y consumo (cada vez más acelerados) de mensajes y significados que irrumpen en las diferentes etapas del proceso. Asistimos de esta manera a una actualización del proceso comunicativo que, si bien evoca aspectos del modelo lineal de producción-circulación-consumo, plantea la existencia de interrelaciones que atraviesan la lógica misma de cada una de las etapas de este proceso, planteando la pregunta sobre los puntos de intersección (y por tanto de encuentro, significación, resignificación y creación) en los cuales la mediación (o las hipermediaciones desde la lectura de Scolari) despliega su potencial creativo de transformación desde los propios sujetos.

En un segundo grupo se encuentran las categorías asociadas a la producción de sentido, la mediación y la cultura, que emergen a finales de los años setenta desde una perspectiva latinoamericana de la comunicación,

Fue entonces cuando, de la mano de autores regionales como Jesús MartínBarbero, José Joaquín Brunner, Renato Ortiz y Néstor García-Canclini, surgieron conceptos como mediación e hibridación, que transformaron la manera como se entiende la comunicación, el papel de los medios, su relación con las dinámicas culturales y los vínculos entre cultura popular y cultura masiva. Esa Escuela Latinoamericana nunca se cerró sobre sí misma sino que entabló un diálogo permanente con tradiciones intelectuales de otros lugares, sin perder de vista su especificidad (Valencia R., 2012, pág. 157).

Los marcos de investigación en estos campos, por lo tanto, retoman de manera importante a estos autores, sus apuestas epistemológicas y sus abordajes de la comunicación centrados en la mediación, la producción de sentido y la cultura.

Vinculado a la Escuela Latinoamérica de la Comunicación, este grupo de categorías reconoce entonces la capacidad de significación y resignificación (producción de sentido) que los sujetos hacen de los contenidos producidos por los medios de comunicación. Las tres categorías -al igual que los procesos de producción, circulación y consumo- se entienden solo a partir de sus interrelaciones, pues los procesos de mediación implican procesos de producción de sentido que pasan por entramados culturales y por emergencia de nuevas significaciones que impactan en la configuración de las diversas culturas. Sobre estas relaciones se encuentran perspectivas diversas, por ejemplo, el análisis de la mediación desde las instituciones y los medios (Martín Serrano, 2011); la mediación como posibilidad de transformación y reinvención de la realidad (Barbero, 2012) y como resignificación de esa realidad a partir de las producciones que se dan a través de las tecnologías de la comunicación, del uso de los medios de comunicación tradicionales y

\footnotetext{
$4 \quad$ Los prosumidores. Según Islas (2010), es un concepto introducido por Alvin Toffler en su libro "La tercera ola" en el año 1981 y se refiere a quienes crean bienes, servicios o experiencias para su propio uso o disfrute, antes que para venderlos o intercambiarlos. "Cuando como individuos o colectivos, PROducimos y conSUMIMOS nuestro propio output, estamos prosumiendo" Toffler y Toffler (2006), citado por Islas (2010), pág. 51.
} 
de los interacciones producto del consumo y del mercado, entre otras (García Canclini E Villoro, 2013).

Continuando con el grupo de categorías identificadas, se pueden citar otras en las que también se evidencian convergencias; Lenguajes, estéticas y narrativas que constituyen un conjunto de expresiones vinculadas a las discursividades, las visualidades, las formas de ser y representar el mundo a partir de las escritas, las oralidades o el uso de las imágenes. Este conjunto de categorías se encuentra vinculado, por tanto, a las formas de expresión de los individuos y los grupos, y cómo recurren a diversos lenguajes y formas de narrar que permiten la configuración de su realidad (Scolari, 2013) y (Ricouer, 2006).

Por otro lado, están Soportes, plataformas y artefactos. Aquí se encuentra propiamente el ámbito de la producción. Hace referencia a los artefactos comunicativos producidos por diseñadores ${ }^{5}$ (Krippendorff, 2011), comunicadores y publicistas y a las herramientas, dispositivos y plataformas privilegiadas para determinados tipos de producción y, desde luego, los medios masivos, alternativos, tradicionales, etc.

Por otro lado se encuentra el grupo Gestión y estrategias de comunicación, en donde las estrategias de comunicación son procesos comunicativos de diseño e intervención de problemáticas específicas y no solo en términos de producción y transmisión de información. La comunicación y la gestión estratégica hacen relación entonces a soluciones que se brindan desde la comunicación (Preciado-Hoyos \& Guzmán-Ramírez, 2012), ya sea visual, publicitaria, política, organizacional, estratégica, alternativa o para el cambio social, entre otras, en aras de resolver un problema o necesidad o transformar una situación (Massoni, 2011), (Scheinsohn, 2010). También incluye la gestión de proyectos, gestión de la comunicación, gestión de medios, gestión de agendas, etc.

Como categoría evidente desde el ejercicio práctico, en mayor medida que desde el epistemológico, se encuentra la creatividad, en este caso, la creatividad aplicada que es una herramienta fundamental de los campos profesionales de la comunicación, la publicidad, el mercadeo y el diseño gráfico, puesto que éstos recurren a la creatividad como un recurso indispensable en el desarrollo e implementación de estrategias dirigidas al posicionamiento de productos o de iniciativas organizacionales o institucionales "donde el eje central del trabajo a desarrollar exige un componente importante de creación, puesto que sus productos han de ser permanentemente nuevos, y porque precisamente gran parte de su éxito recae directamente sobre la mayor o menor creatividad que comporten" (Corbalán Berná, 2008, pág. 18). Adicionalmente, existe también un enorme potencial para pensar desde los campos mencionados el papel de la creatividad en procesos deliberados de transformación social y cultural (Cuesta \& Lora, 2015).

Finalmente, la última categoría convergente es la de Públicos que implica a todas las personas, colectivos, comunidades a los que se dirige la comunicación, ya sea visual,

\footnotetext{
A este respecto la teoría artificialidad desarrollada por Krippendorff (2011), resulta ser un importante referente, al plantear que los artefactos son más que elementos tangibles, para este autor, la noción de artefactos comprende productos, bienes, servicios e identidades, interfaces, sistemas multiusuarios, redes, proyectos y discursos.
} 
publicitaria, estratégica, social, organizacional, etc. Por ejemplo, para los medios masivos son audiencias, para la publicidad son el target, para la comunicación estratégica son stakeholders (Capriotti, 2009).

\section{Algunas pistas para comprender las categorías convergentes desde el concepto de creatividad}

Ahora bien, además de los grupos de categorías identificadas y señaladas anteriormente, se quiere centrar la atención en la categoría creatividad, con el ánimo de ilustrar cómo funcionan estas convergencias en los campos planteados en este estudio. La creatividad se ha convertido en un punto importante de reflexión en el interior de los procesos que adelanta la Facultad de Ciencias de la Comunicación, y desde los campos profesionales de la comunicación, el diseño gráfico y la publicidad, puesto que estos recurren a la creatividad con el fin de transformar lo previo a partir de la selección, del redescubrimiento, la resignificación, la reformulación y la combinación de los elementos ya existentes: el comunicador creativo -sea comunicador social, periodista, diseñador gráfico o publicista- no tiene en la reproducción de la realidad circundante su fin último, pues su ejercicio consiste en anticiparse a los acontecimientos, en apreciar la realidad como quien observa todos los lados de un cubo, en arriesgarse a soluciones no ensayadas.

Desde el campo de la comunicación social, la creatividad emerge como una categoría útil para pensar las producciones culturales a partir de la resignificación de realidades sociales y comunicativas y de la redistribución igualmente creativa de esas producciones a través de nuevos circuitos mediados por las tecnologías de la comunicación, de la convergencia de nuevas narrativas y plataformas y/o de la innovación en el uso de los medios de comunicación tradicionales y de los circuitos de consumo y del mercado (García Canclini \& Villoro, 2013). En este sentido, pensar la creatividad en la comunicación permite entonces ampliar la comprensión de las prácticas comunicativas, pero sobre todo acerca del uso creativo que tienen las personas de los contenidos mediatizados y su capacidad para resignificarlos al usar otros referentes o marcos de sentido diferentes a los utilizados por los emisores primarios, especialmente, los medios masivos. Igualmente, implica entender que dicha mediación adquiere grados de complejidad, mayores si comprendemos que esos procesos de resignificación y producción se han transformado, y de manera significativa, con la incursión de las tecnologías de la información y la comunicación.

En el caso del campo del diseño gráfico, la creatividad se entiende como la capacidad de encontrar soluciones a los problemas que plantea la comunicación visual y, por tanto, se encarga de la creación y producción de artefactos simbólicos que buscan la solución de dichos problemas comunicativos. Su estudio enfocado en el producto, el proceso y las características personales del creativo, busca desde el ámbito de la imagen herramientas de mediación y transformación vinculadas a lo estético, lo artístico y la innovación.

En el ámbito de la publicidad y el mercado, la creatividad se orienta no solo hacia la persuasión del destinatario, entendida esta como un proceso de influencia en el que intervienen la incitación, la intencionalidad, la seducción, la razón y la emoción, sino como 
capacidad de crear nuevos modos de acercar al consumidor con la industria publicitaria en donde "la publicidad debe ser divertida, entretenida, inteligente, pero sobre todo capaz de establecer una especie de juego, trazado en la narrativa de la publicidad, para establecer una nueva relación, un nuevo diálogo entre los anunciantes y los consumidores" (Villadiego P., 2013, pág. 133). La creatividad publicitaria se vincula de esta manera a la comunicación persuasiva y a la comunicación creativa, como propósito, siendo la efectividad en la construcción de mensajes su potencial para transformar procesos de mercado y de agenciar productos y estrategias dirigidas a la solución de problemas.

Por su parte, el mercadeo establece la discusión por las industrias creativas y culturales desde la óptica del intercambio no solamente desde el capital económico sino desde el social, cultural y simbólico. En el mercadeo se encuentra la clave para entender la innovación bajo la mirada explorativa de la creatividad.

La creatividad aplicada y ahora, lo que se podría denominar "comunicación creativa", en los tres casos, ha venido cumpliendo un papel fundamental en la resignificación de marcos culturales con propósitos distintos. La comunicación estratégica, la publicidad, el mercadeo y el diseño gráfico, todos vinculados a las ciencias de la comunicación, cuentan con una trayectoria importante desde las prácticas profesionales al recurrir a la creatividad como un recurso indispensable en el desarrollo e implementación de estrategias dirigidas al posicionamiento de productos o de iniciativas organizacionales o institucionales, y en consecuencia, se convierte en una categoría validada desde la producción misma de la Facultad de Ciencias de la Comunicación, desde donde se extraen estos resultados, a partir de la relación interdisciplinaria en los tres escenarios analizados.

En este punto, reconocer la creatividad, no como categoría estática (Cuesta \& Lora, 2015), sino como un concepto "nómada o "viajero", o "convergente" y en proceso de construcción a partir de sus ámbitos de aplicación, de sus relaciones y usos, los procesos curriculares y las apuestas de investigación -como en el caso de la Facultad de Ciencias de la Comunicación y sus tres programas- no buscan apenas reproducir las concepciones disciplinares sobre la creatividad, sino comprender lo que la creatividad "puede hacer" -siguiendo en este punto la propuesta metodológica de Bal (2012)- problematizando sus usos y potencialidades.

Por lo tanto, más que reconocer su dimensión estática y representacional, habitualmente asociada a la creatividad aplicada y a la producción de artefactos comunicativos, la creatividad, comprendida también como mediación creativa (Cuesta $E$ Lora, 2015), ofrece un camino metodológico para entender cómo dicha creatividad apuesta (o no) a transformaciones sociales y comunicativas en diferentes ámbitos de la vida social.

En consecuencia, la convergencia conceptual en torno a la creatividad en los campos de la comunicación social, el periodismo, el diseño gráfico, la publicidad y el mercadeo permite entonces visualizar nuevos escenarios de investigación que indaguen - desde el su ámbito de aplicación y no apenas desde los referentes teóricos y epistemológicos de diversas disciplinas- cómo "opera" la creatividad en la reconfiguración y transformación de realidades sociales y comunicativas 


\section{Metodología}

A partir de las categorías enunciadas anteriormente, se toman como referencia los artículos de investigación y los proyectos de los dos Grupos de Investigación actualmente vinculados a la Facultad de Ciencias de la Comunicación: Grupo Comunicación, Cultura y Tecnología (creado en el año 2004) y Grupo Nipón Estudio Ánime (creado en el año 2009), durante el periodo 2012-2016 y también los trabajos de grado preparados por los estudiantes como opción de grado, para determinar a cuál grupo de categorías se vinculan según su objeto de indagación, a fin de clasificarlas y determinar si responden o no a dichos grupos. Es de anotar que un mismo trabajo, proyecto o artículo pueden responder a varias categorías.

De esta manera, este estudio partió de una revisión de 313 documentos de la Facultad de Ciencias de la Comunicación, durante el periodo 2012-2016, bajo un enfoque mixto. Este estudio analítico-descriptivo implicó el análisis de contenido de 67 artículos de investigación publicados por investigadores, 46 proyectos de investigación y 198 trabajos de grado elaborados por estudiantes y se contrastó la información de dichos documentos a fin de validar empíricamente los siete grupos de categorías previamente identificadas donde se considera existen convergencias en campos de la comunicación, el diseño, la publicidad y el mercadeo.

\section{Resultados}

\section{Artículos de investigación de los dos grupos de investigación de la Facultad}

Como se observa en las gráficas 1 y 2, se hace relevante la producción en la categoría "Producción de sentido, mediación y cultura", seguida de la categoría "Producción, circulación y consumo". La categoría "Lenguajes, estéticas y narrativas" es relevante para el Grupo de Comunicación, Cultura y Tecnología dado que la naturaleza del grupo, el tiempo de trayectoria y las líneas planteadas permiten desarrollar mayores productos alrededor de dicha categoría. Como se observa, las categorías creatividad y públicos tienen poca relevancia dentro de este conjunto de resultados.

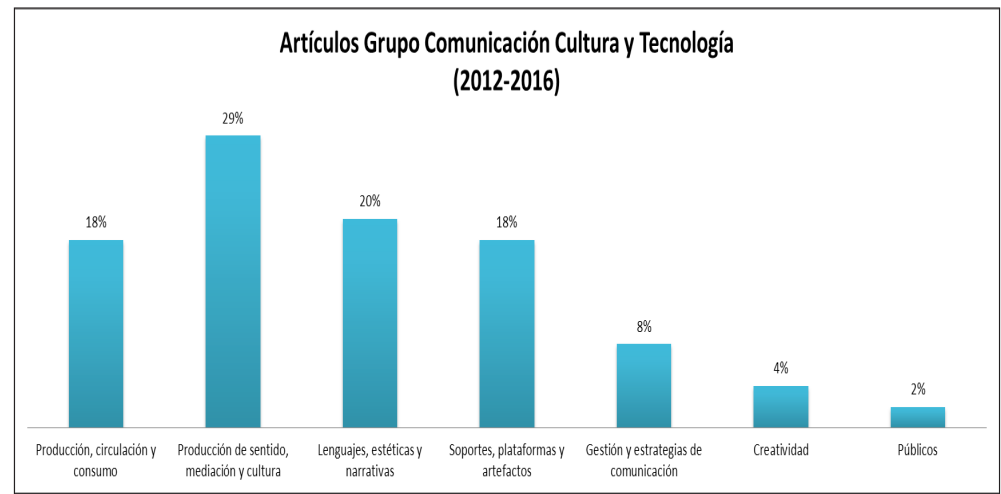

Gráfica 1. Artículos del Grupo Comunicación, Cultura y Tecnología (2012-2016).

Fuente: elaboración propia 


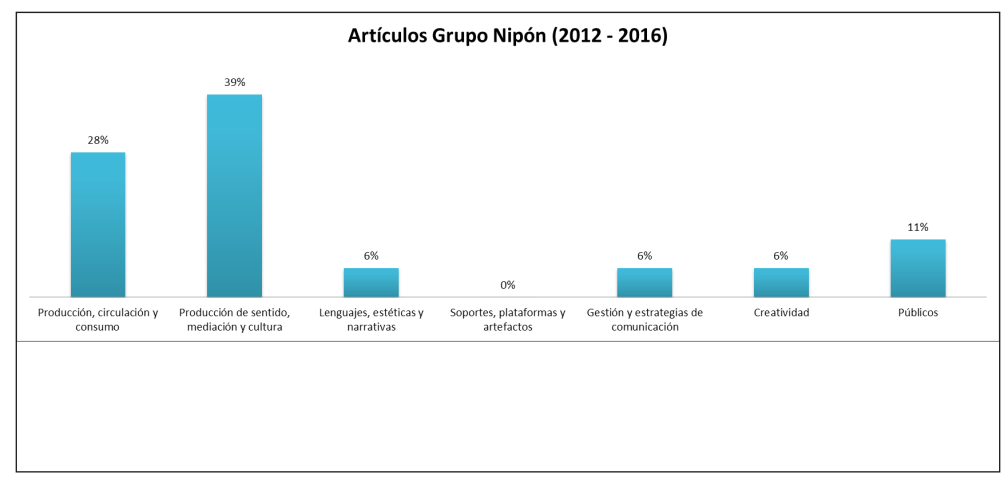

Gráfica 2. Artículos del Grupo Nipón Estudio Ánime (2012-2016).

Fuente: elaboración propia

\section{Proyectos de investigación de los dos grupos de investigación de la Facultad}

Para el caso de los proyectos de la gráfica 3, coincide la relevancia de las categorías "Producción, circulación y consumo" y "Producción de sentido, mediación y cultura", dado que los proyectos desarrollados por el grupo han seguido la línea de investigación con el mismo nombre del Grupo, "Comunicación, Cultura y Tecnología". Por su parte, en el caso de la gráfica 4, cobra importancia la categoría "Gestión y estrategias de comunicación", dado que la mayoría de los proyectos desarrollados plantean soluciones problemáticas sociales, ya sea a través del diseño de estrategias de comunicación, didácticas o pedagógicas o a través del análisis propio de procesos mediante la formulación de diagnósticos.

Esta última gráfica consolida la información de los trabajos de grado desarrollados por los estudiantes de la Facultad durante el periodo de análisis. Se observa que la categoría que reporta mayor relevancia es "Producción, circulación y consumo", lo que es coincidente con los resultados de proyectos y artículos; este grupo de categorías se instala como un aspecto de importancia para la investigación de la Facultad. En un segundo nivel se encuentran dos categorías "Lenguajes, estéticas y narrativas", donde al parecer trabajos de grado que se preocupan por el análisis de los discursos, los diferentes tipos de lenguaje ya sea del campo del diseño, de la comunicación o de la publicidad, resultan de relevancia en las investigaciones realizadas por estudiantes. En un tercer nivel de importancia se encuentran los "Soportes, plataformas y artefactos", seguidos de la "Gestión y estrategias de comunicación", las cuales se explican por el hecho de que las investigaciones de los estudiantes de los tres programas en parte responden a la creación de soportes (revistas, plataformas web, productos multimediales, programas radiofónicos, diseño de contenidos a través de diferentes medios) o plantean el diseño de soluciones a través del análisis de situaciones y la formulación de diversas estrategias ya sea desde la comunicación visual, la comunicación social, la comunicación para el cambio social, la comunicación organizacional, la publicidad o el mercadeo. 


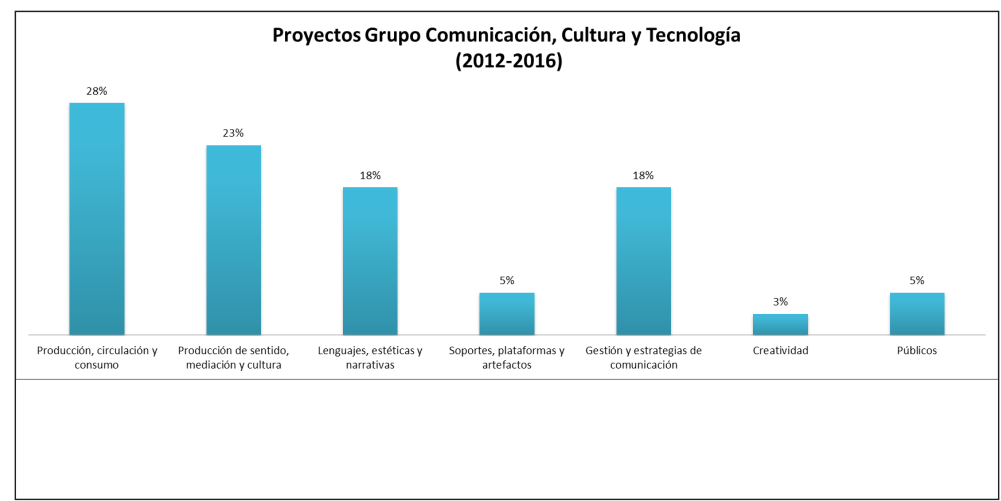

Gráfica 3. Proyectos del Grupo Comunicación, Cultura y Tecnología (2012-2016).

Fuente: elaboración propia

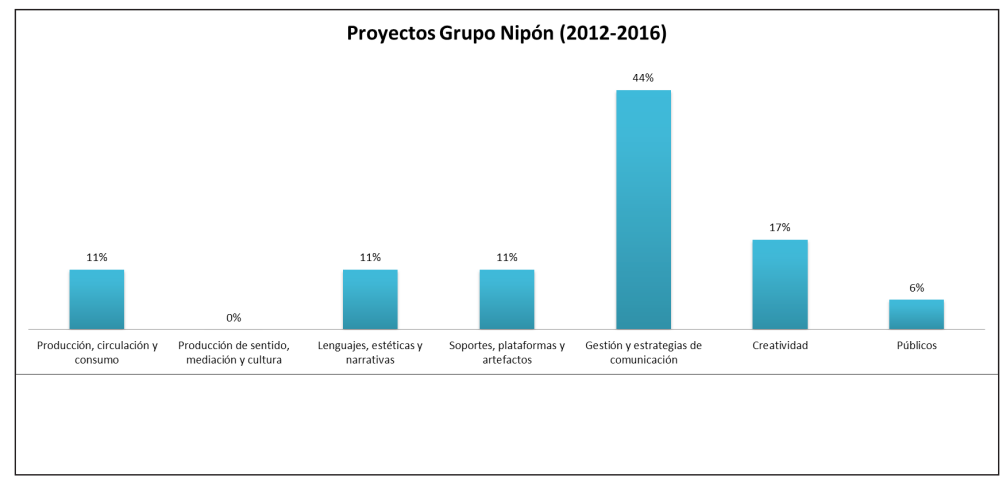

Gráfica 4. Proyectos de Investigación del Grupo Nipón Estudio Ánime (2012-2016).

Fuente: elaboración propia

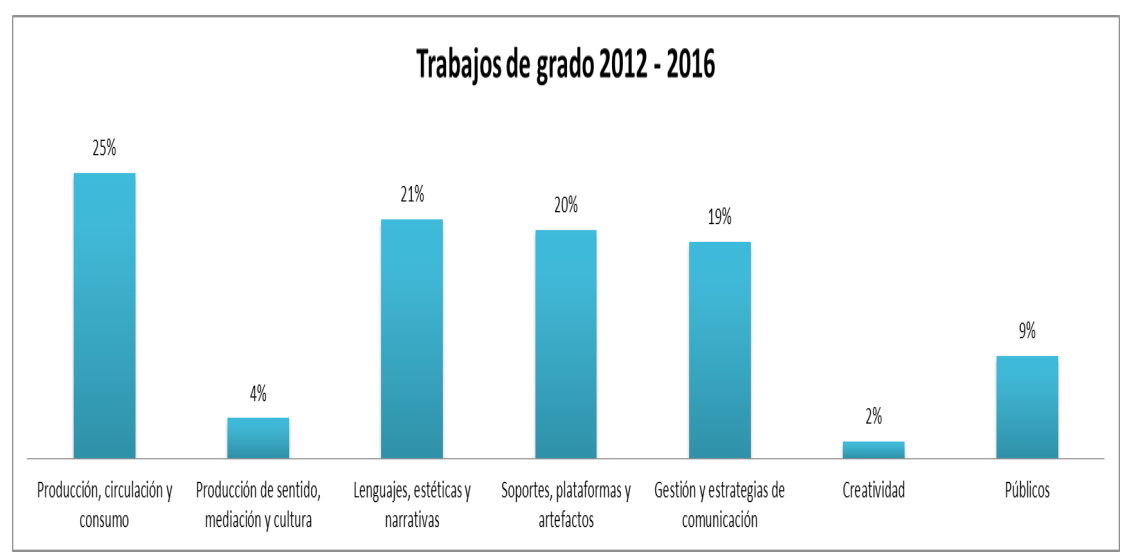

Gráfica 5. Trabajos de grado (2012-2016).

Fuente: elaboración propia 


\section{Conclusiones}

De acuerdo con los resultados obtenidos se puede constatar que los conceptos interdisciplinares y convergentes más importantes a los campos de la comunicación, la publicidad, el diseño y el mercado, a partir de los documentos analizados, fueron producción, circulación y consumo, con una frecuencia de $11,5 \%$; lenguajes, estéticas y narrativas, con $8,83 \%$ y gestión y estrategias de comunicación con $7,71 \%$. Aunque la categoría creatividad tuvo menor relevancia $(3,28 \%)$, desde el análisis planteado en apartados anteriores, también se pudo demostrar que es un concepto que ha venido atravesando los campos analizados.

De los resultados encontrados se puede destacar un hallazgo compartido con el trabajo de Vázquez-Alonso \& Manassero-Mas (2017), en tanto que los conceptos disciplinares suelen "emplear diferentes denominaciones en la literatura, aunque semánticamente sean equivalentes" (pág. 29); por ejemplo, los públicos, receptores, grupos de interés o audiencias cobran nuevos significados dependiendo del campo o del contexto, pero en su esencia o naturaleza conceptual no se modifican, es decir, mantienen su equivalencia semántica.

Por otra parte, este recorrido ha permitido demostrar tanto desde las relaciones conceptuales como desde la evidencia empírica, la existencia de conceptos interdisciplinares, en este caso, comunes a los campos de la comunicación, la publicidad, el diseño y el mercadeo. Esta convergencia conceptual, producto ya sea de la interdisciplinariedad (Tamayo, 2003), de la migración conceptual (Vásquez-Alonso \& Manassero-Mas, 2017), de la historia conceptual interdisciplinar (Oncina, Miravet EVizcaino, 2014) o de la resignificación o transformación que sufren los conceptos a través del tiempo (Bal, 2002), propone un desafío para el campo de la comunicación que consiste en pensarse o repensarse más a partir de las posibilidades de la convergencia, del potencial que muestra la mutabilidad y transversalidad de los conceptos, desde la riqueza que origina en el diálogo entre saberes y desde de la interdisciplinariedad como capacidad de ir más allá de los límites tradicionales de las disciplinas, y menos en las diferencias, las ausencias o la amplitud de elementos que han compuesto el vasto campo de la investigación en la comunicación.

En este sentido, vale la pena reafirmar que frente a la necesidad de resolver problemas complejos tanto para el campo de la comunicación como para otros campos y disciplinas (Vázquez-Alonso \& Manassero-Mas, 2017), la interdisciplinariedad surge como una posibilidad de abordar la realidad desde saberes y conocimientos que logren complementariedad y posibiliten procesos significativos de transformación en la sociedad. El punto de partida para este propósito es comprender que los conceptos ya no son de exclusividad de una disciplina, sino que al dinamizarse, resignificarse y migrar, amplían el horizonte de las disciplinas, las enriquecen y permiten quizá una mirada más holística de la realidad. 


\section{Referencias bibliográficas}

Aguirre, P. d., Anaya, M. d., Laurencio, R. L., \& Casco López, J. (2013). Investigación aplicada e interdisciplinariedad en las ciencias de la comunicación. Prisma Social, 294-320.

Bal, M. (2002). Conceptos viajeros en las humanidades. Toronto: University of Toronto Press.

Barbero, J. M. (2012). De la Comunicacion a la Cultura: perder el "objeto" para ganar el proceso. Signo y Pensamiento(60), 76-84.

Boni, F. (2008). Teorías de los medios de comunicación. Barcelona: Universidad Autónoma de Barcelona, Universitat de Valencia, Universitat Pompeu Fabra.

Capriotti, P. (2009). Los públicos de las organizaciones. En P. Capriotti, Branding Corporativo (pág. 273). Santiago de Chile: Colección de Libros de la Empresa.

Castellanos, A. (2015). Encuentros y desencuentros sobre las teorías de la comunicación, el diseño, la publicidad y el mercadeo. En N. Gualdron, E J. Palacios, Memorias del Primer Encuentro de Investigación en Comunicación (pág. 80). Bogotá: Fundación U. Los Libertadores.

Corbalán Berná, J. (2008). ¿De qué se habla cuando hablamos de creatividad? Cuadernos de la Facultad de Humanidades y Ciencias Sociales. Universidad Nacional de Jujuy (35), 11-21.

Craig, R. T. (1999). Communication theory as a field. Communication Theory, 9 (2), 116-161.

Cuesta, O., \& Lora, P. (2015). La mediación creativaUn acercamiento a su construcción conceptual. Revista Luciérnaga-Comunicación 14 (1) 46-63.

De San Eugenio Vela, J., E Silva Echeto, V. (2014). La investigación en Comunicación ante una encrucijada: de la teoría de los campos a la diseminación y diversidad gnoseológica. Estudio inicial comparado entre España, Brasil y Chile. Palabra clave, 17 (3), 803-827.

Donsbach, W. (2012). The Identity of Communication Research. Signo y Pensamiento, 30 (60), 18-29.

Galindo-Cáceres, J. (2013). Eulalio ferrer y la comunicología. un apunte de vida y obra de un gran maestro del pensamiento en comunicación. Razón y Palabra, 17 (2_83), 69-81.

García Canclini, N., E Villoro, J. (2013). La creatividad redistribuida. México: Siglo XXI Editores.

Igartua, J. J. (2012). Tendencias actuales en los estudios cuantitativos en comunicación. Comunicación $y$ Sociedad (enero-junio), 8 (2), 15-40.

Islas, O. (2010). Internet 2.0: El territorio digital de los prosumidores. Revista Estudios Culturales, 3 (5), 43-63.

Knauf, M. (12 de Nov de 2014). Is the Sustainability Revolution Devouring Its Own Children?Understanding Sustainability as a Travelling Concept and the Role Played by Two German Discourses on Sustainability. Forests (5), 2647-2657.

Krippendorff, K. (2011). Principles of Design and a Trajectory of Artificiality. Journal of Producto Innovation Management, 28 (3), 411-418.

Lancien, T., Cardy, H., Delatte, J., Delavaud, G., Froissart, P., Rodionof, A., y otros. (2004). La investigación sobre la comunicación en Francia. Tendencias y carencias. Comunicación y Sociedad (2), 37-70.

Martín Serrano, M. (2011). Mediación y sociedad. Voz "Mediación". Chasqui. Revista Latinoamericana de 
Comunicación, 24-26. (114-115),

Martínez-Nicolás, M., E Saperas-Lapiedra, E. (2016). Objetos de estudio y orientación metodológica de la reciente investigación sobre comunicación en España (2008-2014). Revista Latina de Comunicación Social (71), 1365-1384.

Massoni, S. (2011). Comunicación estratégica. Comunicación para la innovación. Santa Fe, Argentina: Homo Sapiens.

Neumann, B., E Nünning, A. (2012). Travelling Concepts for the Study of Culture. Boston: De Gruyter.

Olson, G. (2012). Gender as a Travelling Concept: A feminist perspective. En B. Neuman, E A. (. Nünning, Traveling concepts for the study of culture (pág. 417). Boston: De Gruyter.

Oncina, F., Miravet, N., E Vizcaíno, H. (2014). Conceptos nómadas: Auto-determinación. Valencia: Universitat de València.

Otero, E. (2010). Sobre la condición fragmentaria y menesterosa de los estudios en comunicación. Cuadernos de Información (27, julio-diciembre), 7-14.

Palacios, J., Lora, P., Cárdenas, E Guillermo. (2016). Auto-reflexión sobre los procesos internos de investigación: la producción y percepción de los docentes y los estudiantes de la Facultad de Ciencias de la Comunicación. Razón y Palabra, 20 (2_93), 914-925.

Piñuel, J. L. (2009). La comunicación como objeto científico de estudio, como campo de análisis y como disciplina científica. Contratexto Digital (18), 1-22.

Potter, W. J., \& Riddle, K. (2007). A Content Analysis of the Media Effects Literature. Journalism \& Mass Communication Quarterly, 84 (1), 90-104.

Preciado-Hoyos, A., E Guzmán-Ramírez, H. (2012). Gestión de la comunicación estratégica en los sectores empresarial, de desarrollo y público. Estudio comparativo. Palabra Clave, 15 (1), 128-159.

Ricouer, P. (2006). Tiempo y narración I. México: Siglo XXI Editores.

Roncallo-Dow, S. (2013). Entre la interdisciplinariedad, las epistemologías y los objetos. Palabra Clave, 16 (2), 269-281.

Scheinsohn, D. (2010). Comunicación estratégica. Cuadernos del Centro de Estudios en Diseño y Comunicación. Ensayos (33), 17-22.

Scolari, C. (2013). Narrativas transmedia:cuando todos los medios cuentan. Barcelona: Planeta.

Sierra-Gutiérrez, L. I. (Marzo de 2016). La paradójica centralidad de las teorías de la comunicación. Palabra Clave, 19 (1), 15-56.

Tamayo, M. (2003). El proceso de la investigación científica. Ciudad de México: Limusa S. A.

Vázquez-Alonso, Á., E Manassero-Mas, M.-A. (2017). Interdisciplinariedad y conceptos nómadas en didáctica de la ciencia: consecuencias para la investigación. Revista Eureka sobre Enseñanza y Divulgación de las Ciencias, 14 (1), 24-37.

Vidales G., C. (2015). Historia, teoría e investigación de la comunicación. Comunicación y Sociedad (23), $11-43$.

Villadiego P., M. (2013). Narrativas del juego de la publicidad televisiva. Elementos para una comprensión en perspectiva lúdica. Signo y Pensamiento, 32 (62), 130-148. 
Vizcarra, F. (2007). Estudios sobre comunicación en Baja California: Tendencias de investigación y análisis 1943-2005. Global Media Journal, 4 (7), 1-12.

Vizer, E. A., E Carvalho, H. (2015). La perspectiva ecológica y la hipermediatización socia. Palabra Clave, 18 (4), 1087-1110.

Zicavo, E. (2009). Globalización, mercado y consumos culturales Entrevista a Néstor García Canclini. Cuadernos del CLAEH, 32 (98), 89-96. 